\title{
GROUP PRESENTATIONS AND FORMAL DEFORMATIONS $\left({ }^{1}\right)$
}

\author{
BY
}

\section{PERRIN WRIGHT}

ABSTRACT. Formal deformations (expansions and collapses) of dimension $\leq 3$ among 2-dimensional polyhedra are explained in terms of a certain collection of operations on finite group presentations. The results are valid for any simple homotopy type of 2-dimensional polyhedra, and simplifications are possible within the simply connected simple homotopy types.

1. Introduction. The relationship between finite group presentations and finite 2-dimensional polyhedra is in evidence at various places in the literature. Furthermore, the folklore has it that there exists a correspondence from the category of finite group presentations and certain operations thereon, to the category of 2-polyhedra and 3-dimensional formal deformations (expansions and collapses). The purpose of this paper is to give a precise formulation of the problem, with solutions, thereby exonerating the folk. The references listed here, with the exception of [2], are articles in which this problem has been addressed to some extent.

Whitehead showed [5] that any two $n$-polyhedra having the same simple homotopy type are formally equivalent under deformations of dimension $n+$ 1, provided $n>2$. For $n=2$, one must apparently deform through 4-dimensional polyhedra. The reducibility of the dimension of the deformation to three is equivalent to the group theoretic problem to be described here.

The author is greatly indebted to the referee for suggesting a proof of Lemma 2 and for contributing substantially to $\$ 8$.

2. The complexes. We shall initially restrict our attention to a special class $C$ of 3-dimensional $C W$-complexes, defined as follows: If $X \in C$, then:

(1) $X^{(0)}$ consists of a single 0 -cell $v$.

(2) $X^{(1)}$ is the union of $X^{0}$ and a finite collection $\left\{x_{i}\right\}$ of 1-cells whose boundaries are attached to $v$.

(3) $X^{(2)}$ is obtained from $X^{(1)}$ by attaching to $X^{(1)}$ a finite collection

Received by the editors December 20, 1973 and, in revised form, April 17, 1974. AMS (MOS) subject classifications (1970). Primary 57C10; Secondary 20F05.

$\left({ }^{1}\right)$ This research was supported by COFRS, Florida State University. 
$\left\{e_{i}\right\}$ of 2-cells, where $\mathrm{Bd} e_{k}$ is subdivided into edges and vertices; each vertex of $\mathrm{Bd} e_{i}$ is sent to $v$ and each (open) edge of $\mathrm{Bd} e_{i}$ is sent either to $v$ or homeomorphically to some (open) $x_{i}$.

(4) $X^{(3)}$ is obtained by attaching to $X^{(2)}$ a finite collection $\left\{d_{i}\right\}$ of 3-cells, where $\mathrm{Bd} d_{i}$ has the structure of a cell complex; each vertex of $\mathrm{Bd} d_{i}$ is sent to $v$, each edge to $v$ or homeomorphically to some $x_{i}$, and each (open) 2-cell homeomorphically to some (open) $e_{i}$, subject to the usual condition that the attaching map be continuous.

3. Elementary expansions and collapses in $C$. An elementary $n$-expansion $K / L$ in $C$ is defined provided $L=K U_{f} B^{n}$, where $f$ attaches (as in 2(4)) to $K$ all of the boundary of $B^{n}$ except one (open) $(n-1)$-cell. An elementary $n$-collapse in $C$ is the inverse of an elementary $n$-expansion, written $L \backslash K$.

There are no 1-expansions or 1-collapses in $C$ because each $K \in C$ has only one vertex.

A formal $n$-deformation from $K$ to $L$ in $C$ is a finite sequence $\left\{K_{0}\right.$, $\left.\ldots, K_{m}\right\}$ in $C$ such that $K_{0}=K, K_{m}=L, K_{i}$ expands or collapses elementarily to $K_{i+1}$, and $\operatorname{dim} K_{i} \leq n$ for all $i$.

4. Presentations. We depart somewhat from the usual definition of a presentation in order to obtain a correspondence between presentations and complexes in $C$. A finite group presentation will herein consist of a set $\left\{x_{i}\right\}$ of distinct symbols, called the generators, together with a set $\left\{r_{i}\right\}$ of distinct symbols, called the relators; $\left\{x_{i}\right\}$ and $\left\{r_{i}\right\}$ shall be indexed by finite subsets of the natural numbers. Associated with each relator $r_{i}$ is a word $\rho_{i}$ (not necessarily reduced) in the generators $\left\{x_{i}\right\}$, and the group presented is the quotient group $F\left\{x_{i}\right\}$ modulo the normal closure of the $\left\{\rho_{i}\right\}$. We shall use the standard notation $\left\{\left\{x_{1}\right\} \mid\left\{r_{i}\right\}\right\}$ for a presentation.

Two presentations $\left\{\left\{x_{i}\right\} \mid\left\{r_{i}\right\}\right\}$ and $\left\{\left\{y_{i}\right\} \mid\left\{s_{i}\right\}\right\}$ will be considered equal if and only if there exist $1-1$ correspondences $\left\{x_{i}\right\} \leftrightarrow\left\{y_{i}\right\}$ and $\left\{r_{i}\right\} \leftrightarrow\left\{s_{i}\right\}$ which preserve the words associated with the relators.

Associated with each presentation $p=\left\{\left\{x_{i}\right\} \mid\left\{r_{i}\right\}\right\}$ is a 2 -complex $K(p)$ $\leqslant C$, unique up to homeomorphism, which is obtained by attaching 1-cells $\left\{x_{i}\right\}$ to $v$, then attaching 2-cells $\left\{e_{i}\right\}$ along their boundaries by the words $\left\{\rho_{i}\right\}$. (If $\rho_{i}$ is the empty word $\varnothing$, then $\partial e_{i}$ is attached to $v$.) Then $\pi_{1} K(p)$ is the group presented by $p$.

Conversely, if $K$ is an oriented 2-complex in $C$, a group presentation $p(K)$ is induced, which is unique up to indexing of the $\left\{x_{i}\right\}$ and $\left\{r_{i}\right\}$ and cyclic permutation of the $\left\{\rho_{i}\right\}$. 
Remark 1. If $i \neq j$, then $r_{i}$ and $r_{j}$ are distinct relators, even if $\rho_{i}=\rho_{j}$. For example, let $p_{1}=\left\{x_{1} \mid r_{1}\right\}$ and $p_{2}=\left\{x_{1} \mid s_{1}, s_{2}\right\}$, where the words associated with $r_{1}, s_{1}, s_{2}$ are all $x_{1}$. Then $p_{1} \neq p_{2}$, and $K\left(p_{1}\right)$ is a 2-cell while $K\left(p_{2}\right)$ is a 2-sphere. We shall generally abuse our notation when no ambiguity is present, and write $p_{2}=\left\{x_{1} \mid x_{1}, x_{1}\right\}$; that is, we shall use $\rho_{i}$ instead of $r_{i}$ in describing the presentation, suppressing (but not forgetting) the indexing of the relators.

Remark 2. Cancellation of adjacent inverses within a relator, taken for granted in word operations, will not be allowed here. For example, if $p_{1}=$ $\{x \mid \varnothing\}$ and $p_{2}=\left\{x \mid x x^{-1}\right\}$, then $K\left(p_{1}\right)=S^{1} \vee S^{2}$ but $K\left(p_{2}\right)$ is a pinched $S^{2}$. We shall show later that cancellation corresponds to a formal 3-deformation.

Remark 3. Each relator word $\rho$ is assumed to be written as a noncollected word in the generators and their inverses; that is, $\rho=x_{1}^{2} x_{2}^{3}$ should be written $x_{1} x_{1} x_{2} x_{2} x_{2}$. In constructing $K(p)$, the corresponding 2-cell may have some boundary edges which are identified to $v$, as long as the remaining edges, taken clockwise from some point, read the word $\rho$. The insertion of edges to be identified with $v$ does not change the homeomorphism type of $K(p)$ and corresponds to insertion of the identity element of $F\left(x_{1}, \ldots, x_{n}\right)$ at various places within the relator word $\rho$.

5. 2-dimensional operations. On a presentation $p=\left\{x_{1}, \ldots, x_{n} \mid r_{1}, \ldots\right.$, $\left.r_{k}\right\}$, define the following operations:

(1) Cyclically permute the letters of any $\rho_{i}$.

(2) Replace $\rho_{i}$ by $\rho_{i}^{-1}$.

(3) Add a generator $\alpha$ and a relator (whose word is) $\alpha w$, where $w$ is a word in $x_{1}, \ldots, x_{n}$ (possibly $\varnothing$ ).

(4) Delete a generator $\alpha$ and a relator $\alpha w$, provided that $\alpha$ does not appear in any other relator or in $w$.

Of these operations, only (3) and (4) alter the homeomorphism type of $K(p)$.

Theorem 1. $K^{2}$ formally 2-deforms to $L^{2}$ in $C$ if and only if $p(K)$ can be transformed to $p(L)$ by operations (1), (2), (3), (4).

Proof. Since there are no 1-deformations in $C$, it suffices to consider a single elementary 2-expansion or collapse. A 2-expansion $K / L$ consists of adding a 1-cell $a$ and a 2-cell $e$ whose boundary is attached via the word $\alpha w$, where $w$ is any word in the 1-cells of $K$. By performing (3) on $p(K)$, followed by (1), (2), if necessary, we obtain $p(L)$. A 2-collapse $K \backslash L$ corresponds to (4); there rnust be a free edge $\alpha$ through which to collapse 
a 2-cell $e$. In $p(K) a$ appears once in the relator $r$ corresponding to $e$, and in no other relator. Say $r=w \alpha w^{\prime}$. Apply (1) to get $\alpha w^{\prime} w$, then (4) to delete the generator $\alpha$ and relator $\alpha w^{\prime} w$. Follow with (1), (2) if necessary.

Conversely, each operation on a presentation $p$ may be realized on $K(p)$ as follows: for (1), (2), do nothing to $K(p)$; for (3), expand; for (4), collapse.

6. The 3-dimensional operation. A 3-deformation between $K^{2}$ and $L^{2}$ in $C$ will be called transient if each 3-expansion is followed immediately by a 3-collapse. There is no accumulation of 3-cells in a transient deformation. Lemina 2 shows that we need devise a presentation operation for transient 3-deformations only.

Lemma 2. If $K^{2}$ 3-deforms to $L^{2}$ in $C$, then $K^{2}$ transiently 3-deforms to $L^{2}$ in $C$.

Proof. Let a 3-deformation $D$ be given. Enumerate the 3-cells $E_{1}$, $\ldots, E_{n}$ in the order in which they appear in $D$, and let $F_{i}$ denote the face through which $E_{i}$ is eventually collapsed.

Construct a transient deformation $D^{\prime}$ in the following manner. When $E_{1}$ is attached in $D$, let it be attached in $D^{\prime}$ but immediately collapsed via $F_{1}$. When $E_{2}$ is attached in $D$, let it be attached in $D^{\prime}$ such that any faces which were attached to $F_{1}$ in $D$ are now subdivided and attached to $\partial E_{1}-$ $\stackrel{\circ}{F}_{1}$ instead, via some map $\phi_{1}$ induced by $F_{1} \subset E_{1} \searrow \partial E_{1}-\stackrel{\circ}{F}_{1}$. The face $F_{2}$ must now be free: this fails only if, in $D, F_{1} \subset \partial E_{2}$ and $F_{2} \subset \partial E_{1}$, which is impossible since it would block the collapse of both $E_{1}$ and $E_{2}$ in $D$. Collapse $E_{2}$ via $F_{2}$.

In a similar fashion, let each subsequent 3-cell $E_{i}$ be attached in $D^{\prime}$ via the composition of its attaching map in $D$ and the maps $\phi_{i-1}, \ldots, \phi_{1}$, then collapsed immediately via $F_{i}$, which must be free or else there would exist a circle of inequalities $F_{i} \subset \partial E_{k_{1}}, F_{k_{1}} \subset \partial E_{k_{2}}, \ldots, F_{k_{m}} \subset \partial E_{i}$, blocking the collapses of $E_{i}, E_{k_{1}}, \ldots, E_{k_{m}}$ in $D$.

We shall now describe an operation on a presentation $\left\{x_{i} \mid r_{i}\right\}$ which corresponds to an elementary transient 3-deformation $K^{2} / H^{3} \succ L^{2}$.

A word $\rho$ in $x_{1}, \ldots, x_{n}$ will be called allowable if it is obtained by the following steps:

(0) Beginning with the empty word, successively insert words of the form $x x^{-1}$ or $x^{-1} x$ at any point in the word, where $x$ is any generator. Call this word $s$. 
(1) Choose any relator $r_{i}$, and let $\rho_{i}^{\prime}$ be any cyclic permutation of $\rho_{i}$. Let $s^{\prime}$ be any cyclic permutation of $s$.

(2) Form the product $s^{\prime} \rho_{i}^{\prime}$.

(3) Optionally perform any cancellations induced by juxtaposition of $s^{\prime}$ and $\rho_{i}^{\prime}$.

(4) Call the new word $s$ again, and iterate steps (1), (2), (3).

operation (5). If $\rho$ is an allowable word in $\left\{x_{i}\right\}$ and if $r_{*}$ is some relator which is used exactly once in constructing $\rho$, change $\rho_{*}$ to $\rho^{-1}$.

To see that (5) corresponds to an elementary transient 3-deformation, list the relators in the order in which they were used in constructing $\rho$, say $r_{i}, \ldots, r_{i} \cdot$

In $S^{2}$, construct a tree $t$ whose edges read counterclockwise (from $s^{2}-t$ ) the word $s$ of step (0). Construct a 2-cell $e_{i}$, whose boundary edges read $\rho_{i}^{\prime}$ and whose intersection with $t$ corresponds to the cancellations (if any) in step (3), so that the word read counterclockwise from $S^{2}-$ $\left(t \cup e_{i_{1}}\right.$ ) is the word $s$ of step (4). (If $\rho_{i_{1}}=\varnothing$, let $e_{i_{1}}$ have one boundary edge labelled $v_{\text {.) }}$

Modelling on operation (5) we construct in this fashion a collapsible cell complex $t \cup e_{i_{1}} \cup \ldots \cup e_{i_{r}}$ in $S^{2}$, and the word read counterclockwise from the complement is the wo!d $\rho$. Let $e$ denote the complementary 2-cell; its clockwise boundary word is $\rho^{-1}$.

Attach $B^{3}$ to $K(p)$ using this subdivision of $S^{2}$, by mapping each vertex of $S^{2}$ to $v$, each edge to the 1-cell or vertex of $K(p)$ whose letter it bears, and each open 2-cell $e_{i} \cdot$ homeomorphically to its counterpart in $K(p)$. This move is an elementary expansion, of which $e$ is the free face.

Let $e_{*}$ be the 2-cell of $K(p)$ corresponding to $r_{*^{*}}$ Since $r_{*}$ was used exactly once in constructing $\rho, e_{*}$ is a free face of the new 3-cell. Collapse the 3-cell via $e_{*^{*}}$. This transient 3-deformation realizes operation (5).

Conversely, consider a 3-deformation $K \nearrow K \cup, f^{3} \backslash L$. Let $p(K)=$ $\left\{\left\{x_{i}\right\} \mid\left\{r_{i}\right\}\right\}$. The expansion is accomplished by attaching $S^{2}-\stackrel{\circ}{e}$ to $K$, where $e$ is some 2-cell in some cell subdivision of $S^{2}$. Let $\rho^{-1}$ be the word read clockwise from $\mathrm{Bd} e$. Then $S^{2}-\stackrel{e}{e}$ is a collapsible complex whose boundary word (read counterclockwise from $e$ ) is $\rho$. Collapse the 2-cells of $s^{2}-e$ in any order and let $t$ be the remaining tree. Each edge of $t$ is mapped by $f$ to some 1-cell $x_{i}$ of $K$. Expand from any vertex of $t$ to $t$ itself; this induces step (0) of operation.(5). The 2-expansion $t \nearrow S^{2}-\stackrel{\circ}{e}$ induces steps (1), (2), (3) for each 2 -cell in the expansion. As a result, the word $\rho$ is 
built up in an allowable fashion from the presentation $p(K)$. If $e_{*}$ is the free face in the collapse $K \cup, B^{3} \searrow L$, it must follow that $r_{*}$ was used exactly once in constructing $\rho$. Apply operation (5) to replace the relator word $\rho_{*}$ by $\rho^{-1}$. This operation realizes the transient 3 -deformation.

The following theorem has now been established.

Theorem 2. $K^{2}$ formally 3-deforms to $L^{2}$ in $C$ if and only if $p(K)$ can be transformed to $p(L)$ by operations (1) through (5).

7. Consequences of the operations. The following operations can be performed as a composition of operations (1)-(5).

Cancellation. Suppose some relator $r$ has associated word $\rho=u x x^{-1} v$, where $u$ and $v$ are words in $\left\{x_{i}\right\}$. We may replace $\rho$ by $u v$ by the operations

$$
\begin{aligned}
\left\{\mid u x x^{-1} v\right\} & \stackrel{1}{\rightarrow}\left\{\mid x x^{-1} w\right\} \stackrel{3}{\rightarrow}\left\{a \mid x x^{-1} w, a x^{-1}\right\} \\
& \stackrel{5}{\rightarrow}\left\{a \mid w^{-1} x a^{-1}, a x^{-1}\right\} \stackrel{5}{\rightarrow}\left\{a \mid w, a x^{-1}\right\} \\
& \stackrel{4}{\rightarrow}\{\mid w\} \stackrel{1}{\rightarrow}\{\mid u v\} .
\end{aligned}
$$

Coriugation. To replace $\rho$ by $g^{-1} \rho g$, where $g$ is any word in $\left\{x_{i}\right\}$, do repeated applications of the sequence $\rho \rightarrow x x^{-1} \rho \rightarrow x^{-1} \rho x$.

Forming products. If $r_{i}, r_{j}$ are relators and $i \neq j$, we may replace $\rho_{i}$ by $\rho_{i} \rho_{j}$ by

$$
\left\{\mid \rho_{i}, \rho_{j}\right\} \stackrel{5}{\rightarrow}\left\{\mid\left(\rho_{i} \rho_{j}\right)^{-1}, \rho_{j}\right\} \stackrel{2}{\rightarrow}\left\{\mid \rho_{i} \rho_{j}, \rho_{j}\right\}
$$

It is necessary for operation (5) that $i \neq j$, to ensure that $r_{i}$ is used exactly once in constructing $\rho_{i} \rho_{j}$

8. Generalization to polyhedra. It is desirable to generalize Theorem 2 to a theorem about polyhedra. The necessary ingredients are the representation of polyhedra by elements of $C$, and the invariance of 3-deformation classes under this representation.

The first generalization is to cell complexes. If $K$ is any cell complex and $T$ is any tree in $K$ which contains all vertices, then $K / T \in C$.

Lemma 3. $K^{2}$ formally 3-deforms (through cell complexes) to $K / T$. If $T_{0}, T_{1}$ are trees in $K$, then $K / T_{0}$ 3-deforms in $C$ to $K / T_{1}$.

Proof. Let $K \times I$ have the product structure. Then $(K \times I) /(T \times 1) \backslash$ $(K \times 1) /(T \times 1) \cong K / T$. Also

$$
(K \times I) /(T \times 1) \backslash(K \times 0) \cup(T \times I) /(T \times 1) \backslash K \times 0,
$$


since $T \backslash 0$. Thus $K$ 3-deforms to $K / T$.

Let $v$ be any vertex of $K$. Let $T=\left(T_{0} \times 0\right) \cup(v \times I) \cup\left(T_{1} \times 1\right)$. Then $K \times I \backslash(K \times 0) \cup T$ by collapsing $K \times I$ vertically to $(K \times 0) \cup\left(T_{1} \times I\right)$, then collapsing $T_{1} \times I$ horizontally to $\left(T_{1} \times 0\right) \cup\left(T_{1} \times 1\right) \cup(v \times I)$. Upon smashing $T$, we obtain $(K \times I) / T \searrow(K \times 0) / T_{0} \cong K / T_{0}$. Similarly $(K \times I) / T$ $\searrow(K \times 1) / T_{1} \cong K / T_{1}$. Since $(K \times I) / T$ has one vertex, $K / T_{0} 3$-deforms in $C$ to $K / T_{1}$.

Lemma 4. Let $K^{2}$ and $L^{2}$ be cell complexes and let $K^{2}$ 3-deform cellularly to $L^{2}$. Let $T$ and $U$ be trees in $K$ and $L$ which contain all vertices. Then $K / T$ 3-deforms in $C$ to $L / U$.

Proof. There is a cell complex $H^{3}$ such that $K^{2} \downarrow H^{3} \backslash L^{2}$. The complex $H^{3}$ is obtained by reordering the 3 -deformation from $K$ to $L$ so that all expansions occur first.

Let $T_{1}=T \cup$ (trail of vertices in $H^{3} \searrow K^{2}$ ) and $U_{1}=U \cup$ (trail of vertices in $H^{3} \searrow L^{2}$ ). Now $T_{1}$ contains no free edges of $H \searrow K$, so this collapse induces a collapse $H / T_{1} \searrow K / T$ in $C$.

Let $X^{2}$ be the 2-complex which remains after collapsing the 3-cells in $H \searrow L$. Then $T_{1} \subset X$, and $H / T_{1} \searrow X / T_{1}$ in $C$. By Lemma 3, $X / T_{1} 3-$ deforms in $C$ to $X / U_{1}$, which in turn collapses to $L / U$ in $C$.

Lemma 5. Let $K^{2}$ be a cell complex and let $K^{\prime}$ be a cell subdivision of $K$. Let $T$ and $T^{\prime}$ be trees in $K$ and $K^{\prime}$ containing all vertices. Then $K / T$ 3-deforms to $K^{\prime} / T^{\prime}$ in $C$.

Proof. Let $K \times I$ have the product structure induced from $K$, except on $K \times 1$ where the structure is induced from $K^{\prime}$. Then $K \cong K \times 0 \downarrow K \times I \searrow$ $K \times 1 \cong K^{\prime}$ as cell complexes, and by Lemma $4, K / T$ 3-deforms to $K^{\prime} / T^{\prime}$ in $C$.

For an arbitrary compact connected 2-polyhedron $P$, a representative $\bar{P}$ of $P$ in $C$ is obtained by triangulating $P$ in any fashion as a cell complex and smashing any tree containing all vertices. A presentation induced by $P$ is any presentation $p(\bar{P})$, where $\bar{P}$ is a representative of $P$ in $C$.

Theorem 3. The following are equivalent:

(i) The polyhedron $P^{2}$ formally 3-deforms (polyhedrally) to the polybedron $Q^{2}$.

(ii) For some representatives $\bar{P}, \bar{Q}$ in $C, \bar{P}$ 3-deforms to $\bar{Q}$ in $C$.

(iii) For all representatives $\bar{P}, \bar{Q}$ in $C, \bar{P}$ 3-deforms to $\bar{Q}$ in $C$.

By virtue of Theorem 2, we obtain 
Corollary 3.1. The polyhedron $P^{2}$ formally 3-deforms to the polyhedron $Q^{2}$ if and only if some (all) induced presentation(s) of $P$ can be transformed to some (all) induced presentation( $s$ ) of $Q$ by operations (1)-(5).

Proof of Theorem 3. (iii) $\rightarrow$ (i). Let $\bar{P}, \bar{Q}$ be representatives of $P, Q$ in C. By Lemma 3, there exist (polyhedral) 3-deformations $P \rightarrow \bar{P}, Q \rightarrow \bar{Q}$, and by hypothesis, $\bar{P}$ 3-deforms to $\bar{Q}$. Hence $P$ 3-deforms polyhedrally to Q.

(i) $\rightarrow$ (ii). If $P^{2} 3$-deforms to $Q^{2}$, there exists a polyhedron $Z^{3}$ such that $P / Z \downarrow Q$. There exist simplicial triangulations $H, K, L$ of $Z, P, Q$ such that $K \swarrow H \backslash L$ simplicially. (These may be obtained by triangulating $Z$, subdividing to get a simplicial collapse to $P$, subdividing further to get a simplicial collapse to $Q$, and invoking [2] to see that the simplicial collapse to $P$ is not lost.) Since $K$ 3-deforms to $L$ simplicially, Lemma 4 states that for any representatives $\bar{P}, \bar{Q}$ of the form $K / T, L / U$ (for these particular $K, L), \bar{P}$ 3-deforms to $\bar{Q}$ in $C$.

(ii) $\rightarrow$ (iii). If $K / T$ and $K^{\prime} / T^{\prime}$ are representatives of $P$ in $C$, then $K$ and $K^{\prime}$ have a common (up to isomorphism) subdivision $K^{\prime \prime}$. Let $T^{\prime \prime}$ be any tree in $K^{\prime \prime}$ containing all vertices. Then by Lemma 5, there are 3-deformations $K / T \rightarrow K^{\prime \prime} / T^{\prime \prime} \rightarrow K^{\prime} / T^{\prime}$ in $C$. Hence if $\bar{P}$ 3-deforms to $\bar{Q}$ in $C$ for some representatives, the same is true for all representatives.

9. Simply connected complexes. If $K \in C$ has $\pi_{1}(K)=1$ then in $p(K)$ $=\left\{x_{1}, \ldots, x_{n} \mid r_{1}, \ldots, r_{k}\right\}$, the normal closure of $r_{1}, \ldots, r_{k}$ in the free group $F\left(x_{1}, \ldots, x_{n}\right)$ is the free group $F\left(x_{1}, \ldots, x_{n}\right)$. With this extra condition, the operations (1)-(5) can be simplified to these:

(0) Cancellation (and its inverse).

(i) Replace $r_{i}$ by $r_{i}^{-1}$.

(ii) Replace $r_{i}$ by $r_{i} r_{j}, i \neq j$.

(iii) Replace $r_{i}$ by $g^{-1} r_{i} g, g \in F\left(x_{1}, \ldots, x_{n}\right)$.

(iv) Add a generator $x$ and a relator $x$.

(v) Delete a generator $x$ and relator $x$ if $x$ appears in no other relator.

It is easily seen that operations (1)-(5) imply the new operations. The converse is also true, and only (3) and (5) present any difficulty. To obtain (3), write $w$ as a product of conjugates of the relators, then add $\{a \mid a\}$ and apply (ii) and (iii) repeatedly to change the relator $a$ to $a w$. To obtain (5), let $w$ be the word built in the process of constructing $\rho$ just prior to the usage of the relator $r_{*}$. Since $w$ is a product of conjugates of the other relators, we can replace $\rho_{*}$ by $w \rho_{*}$. Then $\rho$ is constructed without 
using $r_{*}$ again, so we may replace $w \rho_{*}$ by $\rho$, then $\rho^{-1}$ to get (5).

From the foregoing and Corollary 3.1 we have

Corollary 3.2. $P^{2}$ formally 3-deforms to an n-fold wedge of 2-spheres if and only if all presentations induced by $P$ can be transformed to the presentation with no generators and $n$ empty relators by the operations (0), (i),..., (v).

When $n=0$, this says that contractible 2-polyhedra 3 -deform to a point if and only if their induced presentations can be transformed to the empty presentation $\{\mid\}$ by those operations.

\section{REFERENCES}

1. J. J. Andrews and M. L. Curtis, Free groups and handlebodies, Proc. Amer. Math. Soc. 16 (1965), 192-195. MR 30 \#3454.

2. D. R. J. Chillingworth, Collapsing three-dimensional convex polyhedra, Proc. Cambridge Philos. Soc. 63 (1967), 353-357. MR 35 \# 995.

3. H. F. Trotter, Homology of group systems with applications to knot theory, Ann. of Math. (2) 76 (1962), 464-498. MR 26 \# 761.

4. C. T. C. Wall, Formal deformations, Proc. London Math. Soc. (3) 16 (1966), 342-352. MR 33 \# 1851.

5. J. H. C. Whitehead, Simplicial spaces, nuclei, and m-groups, Proc. London Math. Soc. (2) 45 (1939), 243-327.

6. - On incidence matrices, nuclei, and homotopy types, Ann. of Math. (2) 42 (1941), 1197-1239. MR 3, 142.

DEPARTMENT OF MATHEMATICS, FLORIDA STATE UNIVERSITY, TALLAHASSEE, FLORIDA 32306 